\title{
Leptogenesis with Linear, Inverse or Double Seesaw
}

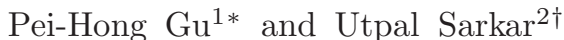 \\ ${ }^{1}$ Max-Planck-Institut für Kernphysik, Saupfercheckweg 1, 69117 Heidelberg, Germany \\ ${ }^{2}$ Physical Research Laboratory, Ahmedabad 380009, India
}

\begin{abstract}
The left-right symmetric model with doublet and bi-doublet Higgs scalars can accommodate linear, inverse or double seesaw for generating small neutrino masses in the presence of three singlet fermions. If the singlet fermions have small Majorana masses, they can form three pairs of quasi-degenerate Majorana fermions with three right-handed neutrinos. The decays of the quasidegenerate Majorana fermions can realize the resonant leptogenesis. Alternatively, the right-handed neutrinos can obtain seesaw suppressed Majorana masses if the singlet fermions are very heavy. In this case leptogenesis, with or without resonant effect, is allowed in the decays of the right-handed neutrinos.
\end{abstract}

PACS numbers: 98.80.Cq, 14.60.Pq, 12.60.Cn, 12.60.Fr

\section{INTRODUCTION}

Neutrinos are massless in the standard model (SM). Under the SM gauge symmetry, one can introduce righthanded neutrinos [1 [5] or Higgs triplet(s) [6] to accommodate the seesaw [1 5] mechanism for generating the small neutrino masses naturally. A more attractive scheme is to consider the left-right symmetric extension of the SM. The left-right symmetric models 7], based on the gauge groups $S U(2)_{L} \times S U(2)_{R} \times U(1)_{B-L}$ have a number of attractive features, such as the natural explanation of weak hypercharge in terms of lepton and baryon numbers, the origin of parity violation, the existence of right-handed neutrinos, etc.. In the original left-right symmetric model [7] with doublet and bi-doublet Higgs scalars, the right-handed Higgs doublet and the Higgs bidoublet are responsible for the left-right and electroweak symmetry breaking, respectively. All of the fermions obtain Dirac masses through their Yukawa couplings with the Higgs bi-doublet. In this sense it is difficult to understand the small neutrino masses.

One possible solution is to replace the Higgs doublets by the Higgs triplets [5]. The right-handed Higgs triplet develops a large vacuum expectation value (VEV) for the heavy masses of the left-handed Higgs triplet and the right-handed Majorana neutrinos. The left-handed neutrinos then can obtain small Majorana masses through the suppressed ratio of the electroweak scale over these heavy masses. In this type-I [1 5] plus II [6] seesaw context, the CP-violation and out-of-equilibrium decays of the right-handed Majorana neutrinos [8] and the lefthanded Higgs triplet 9, 10 can generate a lepton asymmetry through the self-energy [10 12] and vertex corrections [8, 13]. This lepton asymmetry is partially converted to a baryon asymmetry through sphaleron [14] so that we can understand the matter-antimatter asymmetry in the universe. This is the so-called leptogenesis

\footnotetext{
*Electronic address: peihong.gu@mpi-hd.mpg.de
}

†Electronic address: utpal@prl.res.in
[8 13, 15, 17] mechanism.

Alternatively, one can revive the original left-right symmetric model by introducing singlet fermions [18]. These singlet fermions can have Yukawa couplings with the Higgs and lepton doublets. So the seesaw for the small neutrino masses is available in the presence of the Majorana masses of the singlet fermions. Specifically, the small Majorana masses of the singlet fermions will induce the inverse [19] and linear 20] seesaw while the large ones will give the double [18] and linear 20] seesaw. Depending on the size of the Majorana masses of the singlet fermions, the leptogenesis can be realized in two ways: (1) the right-handed neutrinos and the singlet fermions form three pairs of quasi-degenerate Majorana neutrinos; (2) the right-handed neutrinos obtain Majorana masses through the seesaw contributions from the decoupled singlet fermions. We now begin to demonstrate these possibilities in details.

\section{THE MODEL}

For simplicity we do not write down the full Lagrangian. Instead, we only give the part that is relevant for our discussions,

$$
\begin{aligned}
\mathcal{L} \supset & -y \bar{\psi}_{L} \phi \psi_{R}-\tilde{y} \bar{\psi}_{L} \tilde{\phi} \psi_{R}-f_{L} \bar{\psi}_{L} \chi_{L} \xi_{R}-f_{R} \bar{\psi}_{R} \chi_{R} \xi_{R}^{c} \\
& -\frac{1}{2} m_{\xi} \bar{\xi}_{R}^{c} \xi_{R}-\mu \chi_{L}^{\dagger} \phi \chi_{R}-\tilde{\mu} \chi_{L}^{\dagger} \tilde{\phi} \chi_{R}+\text { H.c. }
\end{aligned}
$$

Here $\psi_{L}$ and $\psi_{R}$ denote the left- and right-handed lepton doublets for each family, $\chi_{L}$ and $\chi_{R}$ are the left- and right-handed Higgs doublets, $\phi$ is the Higgs bi-doublet, $\xi_{R}$ stands for the three singlet fermions. We define the left-right discrete symmetry to be the parity, under which the fields transform as

$$
\begin{aligned}
\psi_{L} \equiv(\mathbf{2}, \mathbf{1},-1) & \stackrel{\mathcal{P}}{\longleftrightarrow} \psi_{R} \equiv(\mathbf{1}, \mathbf{2},-1), \\
\chi_{L} \equiv(\mathbf{2}, \mathbf{1},-1) & \stackrel{\mathcal{P}}{\longleftrightarrow} \chi_{R} \equiv(\mathbf{1}, \mathbf{2},-1), \\
\phi \equiv\left(\mathbf{2}, \mathbf{2}^{*}, 0\right) & \stackrel{\mathcal{P}}{\longleftrightarrow} \phi^{\dagger} \equiv\left(\mathbf{2}^{*}, \mathbf{2}, 0\right), \\
\xi_{R} \equiv(\mathbf{1}, \mathbf{1}, 0) & \stackrel{\mathcal{P}}{\longleftrightarrow} \xi_{R}^{c} \equiv(\mathbf{1}, \mathbf{1}, 0) .
\end{aligned}
$$


This constrains $y=y^{\dagger}, \tilde{y}=\tilde{y}^{\dagger}, f_{L}=f_{R}=f, \mu=\mu^{*}$ and $\tilde{\mu}=\tilde{\mu}^{*}$ in Eq. (11).

After the right-handed Higgs doublet $\chi_{R}$ develops its $\operatorname{VEV}\left\langle\chi_{R}\right\rangle$, the $S U(2)_{L} \times S U(2)_{R} \times U(1)_{B-L}$ left-right symmetry is broken down to the $S U(2)_{L} \times U(1)_{Y}$ electroweak symmetry. At this stage, we can conveniently divide the Higgs bi-doublet into two Higgs doublets: one will have a nonzero VEV for the electroweak symmetry breaking and can be identified to the SM one, whereas the other one will not develop any nonzero VEVs but can have a mass of the order of the left-right breaking scale. For this purpose, it is easy to describe

$$
\phi=\left[\phi_{1},-\tilde{\phi}_{2}\right]
$$

and then define

$$
\varphi=\frac{\left\langle\phi_{1}\right\rangle \phi_{1}+\left\langle\phi_{2}\right\rangle \phi_{2}}{\sqrt{\left\langle\phi_{1}\right\rangle^{2}+\left\langle\phi_{2}\right\rangle^{2}}}, \quad \eta=\frac{\left\langle\phi_{1}\right\rangle \phi_{2}-\left\langle\phi_{2}\right\rangle \phi_{1}}{\sqrt{\left\langle\phi_{1}\right\rangle^{2}+\left\langle\phi_{2}\right\rangle^{2}}}
$$

with

$$
\langle\varphi\rangle=\sqrt{\left\langle\phi_{1}\right\rangle^{2}+\left\langle\phi_{2}\right\rangle^{2}}, \quad\langle\eta\rangle=0
$$

for the electroweak symmetry breaking. Thus the righthanded neutrinos $\nu_{R}$ can have the Yukawa couplings to the SM Higgs doublet $\varphi$, given by

$$
\mathcal{L} \supset-h_{\nu} \bar{\psi}_{L} \varphi \nu_{R}+\text { H.c. }
$$

with

$$
h_{\nu}=y \frac{\left\langle\phi_{1}\right\rangle}{\langle\varphi\rangle}+\tilde{y} \frac{\left\langle\phi_{2}\right\rangle}{\langle\varphi\rangle} .
$$

On the other hand, the right-handed neutrinos $\nu_{R}$ and the singlet fermions $\xi_{R}$ mix together as

$$
\mathcal{L} \supset-f\left\langle\chi_{R}\right\rangle \bar{\nu}_{R} \xi_{R}^{c}-\frac{1}{2} m_{\xi} \bar{\xi}_{R}^{c} \xi_{R}+\text { H.c. } .
$$

Clearly, the mass eigenstates, determined by a linear combination of $\nu_{R}$ and $\xi_{R}$, can have very different properties depending on the size of the Majorana mass term $m_{\xi}$. For example, $\nu_{R}$ and $\xi_{R}$ can form three pairs of quasi-degenerate Majorana fermions if $f\left\langle\chi_{R}\right\rangle \gg m_{\xi}$. On the contrary, with $f\left\langle\chi_{R}\right\rangle \ll m_{\xi}$, we can give $\nu_{R}$ Majorana masses due to the seesaw contributions from $\xi_{R}$. In the following we will study the realization of leptogenesis and seesaw in the two limiting cases.

\section{LEPTOGENESIS WITH LINEAR AND INVERSE SEESAW}

In the case with $f\left\langle\chi_{R}\right\rangle \gg m_{\xi}$, we can conveniently choose the base, where $f$ is rotated to be diagonal and real, i.e. $f=\operatorname{diag}\left\{f_{1}, f_{2}, f_{3}\right\}$, and then diagonalize the mass terms (8) by taking the rotations as below,

$$
\begin{aligned}
& \nu_{R_{i}} \simeq \frac{1}{\sqrt{2}}\left(N_{R_{i}}^{+}-i N_{R_{i}}^{-}\right), \\
& \xi_{R_{i}} \simeq \frac{1}{\sqrt{2}}\left(N_{R_{i}}^{+}+i N_{R_{i}}^{-}\right) .
\end{aligned}
$$

Consequently there will be two physical Majorana fermions,

$$
\begin{aligned}
& N_{i}^{+}=N_{R_{i}}^{+}+N_{R_{i}}^{+c}, \\
& N_{i}^{-}=N_{R_{i}}^{-}+N_{R_{i}}^{-c}
\end{aligned}
$$

with

$$
\begin{aligned}
& m_{N_{i}^{+}} \simeq f_{i} v_{\chi}+\frac{1}{2} m_{\xi_{i i}} \\
& m_{N_{i}^{-}} \simeq f_{i} v_{\chi}-\frac{1}{2} m_{\xi_{i i}}
\end{aligned}
$$

The Yukawa couplings (6) can be rewritten as

$$
\mathcal{L} \supset-\frac{1}{\sqrt{2}} h_{\nu_{\alpha i}} \bar{\psi}_{L_{\alpha}} \varphi N_{i}^{+}+\frac{i}{\sqrt{2}} h_{\nu_{\alpha i}} \bar{\psi}_{L_{\alpha}} \varphi N_{i}^{-}+\text {H.c. . }
$$

The singlet fermions $\xi_{R}$ have Yukawa couplings to the left-handed Higgs doublet $\chi_{L}$ [the third term of Eq. (10)]. Furthermore, $\chi_{L}$ mixes with the SM Higgs doublet $\varphi$,

$$
\mathcal{L} \supset-\frac{\left(\mu\left\langle\phi_{1}\right\rangle+\tilde{\mu}\left\langle\phi_{2}\right\rangle\right)\left\langle\chi_{R}\right\rangle}{\langle\varphi\rangle} \chi^{\dagger} \varphi+\text { H.c. . }
$$

So, besides Eq. (12), the Majorana fermions $N^{ \pm}$can have other Yukawa couplings to $\varphi$ by integrating out $\chi_{L}$,

$$
\mathcal{L} \supset-\frac{1}{\sqrt{2}} h_{\xi_{\alpha i}} \bar{\psi}_{L_{\alpha}} \varphi N_{i}^{+}-\frac{i}{\sqrt{2}} h_{\xi_{\alpha i}} \bar{\psi}_{L_{\alpha}} \varphi N_{i}^{-}+\text {H.c. }
$$

with

$$
h_{\xi}=-f \frac{\left\langle\chi_{R}\right\rangle\left(\mu\left\langle\phi_{1}\right\rangle+\tilde{\mu}\left\langle\phi_{2}\right\rangle\right)}{m_{\chi_{L}}^{2}\langle\varphi\rangle} .
$$

Here $m_{\chi_{L}}=\mathcal{O}\left(\left\langle\chi_{R}\right\rangle\right)$ is the mass of $\chi_{L}$. Clearly, we have assumed $m_{\chi_{L}} \gg m_{N_{i}^{ \pm}}$. This assumption is necessary for a successful leptogenesis. For example, $\left\langle\chi_{R}\right\rangle$ should be bigger than $\mathcal{O}\left(10^{7} \mathrm{GeV}\right)$ [9, 21] to guarantee the departure from equilibrium of $N_{1}^{ \pm}$, if $N_{1}^{ \pm}$is the lightest pair with $m_{N_{1}^{ \pm}}=\mathcal{O}(1-10 \mathrm{TeV})$ to account for the generation of a final lepton asymmetry. After the electroweak symmetry breaking, $\varphi$ will develop a $\operatorname{VEV}\langle\varphi\rangle$ and then $\chi_{L}$ will pick up a smaller VEV,

$$
\left\langle\chi_{L}\right\rangle \simeq-\frac{\left\langle\chi_{R}\right\rangle\left(\mu\left\langle\phi_{1}\right\rangle+\tilde{\mu}\left\langle\phi_{2}\right\rangle\right)}{m_{\chi_{L}}^{2}} \simeq-\frac{\mu\left\langle\phi_{1}\right\rangle+\tilde{\mu}\left\langle\phi_{2}\right\rangle}{\left\langle\chi_{R}\right\rangle} .
$$

The Yukawa couplings (15) can simply be given by

$$
\begin{aligned}
h_{\xi} & =-f \frac{\left\langle\chi_{L}\right\rangle}{\langle\varphi\rangle}=-\operatorname{diag}\left\{f_{1}, f_{2}, f_{3}\right\} \frac{\left\langle\chi_{L}\right\rangle}{\langle\varphi\rangle} \\
& =\operatorname{diag}\left\{h_{\xi_{e 1}}, h_{\xi_{\mu 2}}, h_{\xi_{\tau 3}}\right\} .
\end{aligned}
$$




\section{A. Baryon Asymmetry}

Following the standard method [12] of the resonant leptogenesis, we can compute the lepton asymmetry from the decays of each $N_{i}^{ \pm}$,

$$
\begin{aligned}
\varepsilon_{N_{i}^{ \pm}} & =\frac{\sum_{\alpha}\left[\Gamma\left(N_{i}^{ \pm} \rightarrow \psi_{L_{\alpha}}+\varphi^{*}\right)-\Gamma\left(N_{i}^{ \pm} \rightarrow \psi_{L_{\alpha}}^{c}+\varphi\right)\right]}{\sum_{\alpha}\left[\Gamma\left(N_{1}^{ \pm} \rightarrow \psi_{L_{\alpha}}+\varphi^{*}\right)+\Gamma\left(N_{1}^{ \pm} \rightarrow \psi_{L_{\alpha}}^{c}+\varphi\right)\right]} \\
& \simeq \frac{\left(h_{\xi}^{\dagger} h_{\xi}-h_{\nu}^{\dagger} h_{\nu}\right)_{i i} \operatorname{Im}\left[\left(h_{\nu}^{\dagger} h_{\xi}\right)_{i i}\right]}{4 \pi A_{N_{i}^{ \pm}}} \frac{r_{N_{i}}}{r_{N_{i}}^{2}+\frac{1}{64 \pi^{2}} A_{N_{i}^{\mp}}^{2}}
\end{aligned}
$$

with

$$
\begin{aligned}
r_{N_{i}} & =\frac{m_{N_{i}^{+}}^{2}-m_{N_{i}^{-}}^{2}}{m_{N_{i}^{+}} m_{N_{i}^{-}}} \simeq \frac{2 m_{\xi_{i i}}}{f_{i}\left\langle\chi_{R}\right\rangle}, \\
A_{N_{i}^{ \pm}} & =\frac{1}{2}\left[\left(h_{\nu}^{\dagger} \pm h_{\xi}^{\dagger}\right)\left(h_{\nu} \pm h_{\xi}\right)\right]_{i i} .
\end{aligned}
$$

From the calculation (18), we emphasize that the Yukawa couplings (12) and (14) are both necessary ${ }^{1}$ to generate a nonzero lepton asymmetry. For demonstration, we assume $N_{1}^{ \pm}$to be much lighter than $N_{2,3}^{ \pm}$. This means that the final lepton and baryon asymmetry should mainly come from the decays of $N_{1}^{ \pm}$. In the weak and strong washout region, the final baryon asymmetry can be approximately given by [23]

$$
\begin{aligned}
\eta_{B} & =\frac{n_{B}}{s} \\
& =-\frac{28}{79} \times \begin{cases}\frac{\varepsilon_{N_{1}^{ \pm}}}{g_{*}} & \text { for } K_{N_{1}^{ \pm}} \ll 1, \\
\frac{0.3 \varepsilon_{N_{1}^{+}}}{g_{*} K_{N_{1}^{ \pm}}\left(\ln K_{N_{1}^{ \pm}}\right)^{0.6}} & \text { for } K_{N_{1}^{ \pm}} \gg 1\end{cases}
\end{aligned}
$$

with $g_{*} \simeq 106.75$ being the relativistic degrees of freedom (the SM fields). Here the quantity

$$
K_{N_{1}^{ \pm}}=\left.\frac{\Gamma_{N_{1}^{ \pm}}}{2 H(T)}\right|_{T=m_{N_{1}^{ \pm}}}
$$

measures the effectiveness of the decays of $N_{1}^{ \pm}$at the leptogenesis epoch. $\Gamma_{N_{1}^{ \pm}}$and $H(T)$ are the decay width and the Hubble constant, respectively, i.e.

$$
\begin{aligned}
\Gamma_{N_{1}^{ \pm}} & =\frac{1}{8 \pi} A_{N_{1}^{ \pm}} m_{N_{1}^{ \pm}}, \\
H(T) & =\left(\frac{8 \pi^{3} g_{*}}{90}\right)^{\frac{1}{2}} \frac{T^{2}}{M_{\mathrm{Pl}}} .
\end{aligned}
$$

\footnotetext{
${ }^{1}$ In the $S O(10) \rightarrow S U(5) \rightarrow S U(2)_{L} \times U(1)_{Y}$ models for the resonant leptogenesis, one could consider other possibilities [22] to make the right-handed neutrinos and the singlet fermions both having the Yukawa couplings to the SM lepton and Higgs doublets.
}

\section{B. Neutrino Masses}

After the electroweak symmetry breaking, it is easy to read the neutrino mass matrix by making use of the seesaw formula [1 5],

$$
\mathcal{L} \supset-\frac{1}{2} \bar{\nu}_{L} m_{\nu} \nu_{L}^{c}+\text { H.c. }
$$

with

$$
m_{\nu} \simeq h_{\nu} \frac{1}{f^{*}} m_{\xi} \frac{1}{f^{\dagger}} h_{\nu}^{T} \frac{\langle\varphi\rangle^{2}}{\left\langle\chi_{R}\right\rangle^{2}}-\left(h_{\nu}+h_{\nu}^{T}\right) \frac{\langle\varphi\rangle\left\langle\chi_{L}\right\rangle}{\left\langle\chi_{R}\right\rangle} .
$$

The second term is the linear seesaw [20]. As for the first term, it is the inverse seesaw [19] for $f\left\langle\chi_{R}\right\rangle \gg m_{\xi}$. So we denote

$$
m_{\nu}=m_{\nu}^{\text {Inverse }}+m_{\nu}^{\text {Linear }}
$$

\section{Parameter Choice}

For a successful leptogensis and seesaw, we need to choose the parameter space including two types of the Yukawa couplings $\left(h_{\nu}\right.$ and $\left.f\right)$, four $\operatorname{VEVs}\left(\left\langle\chi_{R}\right\rangle,\left\langle\chi_{L}\right\rangle\right.$ and $\left.\left\langle\phi_{1,2}\right\rangle\right)$ and two cubic couplings $(\mu$ and $\tilde{\mu})$.

Firstly, we take

$$
\left\langle\chi_{R}\right\rangle=3 \times 10^{7} \mathrm{GeV}
$$

and then

$$
\begin{aligned}
& m_{N_{1}^{ \pm}}=3 \mathrm{TeV} \text { for } f_{1}=10^{-4} \\
& m_{N_{2,3}^{ \pm}}=30 \mathrm{TeV} \text { for } f_{2,3}=10^{-3} .
\end{aligned}
$$

This choice can guarantee that the gauge interactions of $N_{1}^{ \pm}$have been decoupled at the leptogenesis epoch $T \sim m_{N_{1}^{ \pm}}[9,21]$. Secondly, we assume

$$
\mu \simeq \tilde{\mu}=\mathcal{O}(0.1)\left\langle\chi_{R}\right\rangle
$$

and then perform

$$
\left\langle\chi_{L}\right\rangle=0.1\langle\varphi\rangle
$$

From the above ratio, it is easy to solve

$$
\langle\varphi\rangle \simeq 173 \mathrm{GeV}, \quad\left\langle\chi_{L}\right\rangle \simeq 17 \mathrm{GeV}
$$

for

$$
\sqrt{\langle\varphi\rangle^{2}+\left\langle\chi_{L}\right\rangle^{2}} \simeq 174 \mathrm{GeV}
$$

With the inputs (29) and (31), Eq. (17) induces

$$
h_{\xi_{e 1}}=0.1 h_{\xi_{\mu 2}}=0.1 h_{\xi_{\tau 3}}=-10^{-5} .
$$

Thirdly, we consider

$$
m_{\xi_{i j}}=\mathcal{O}(0.1-1 \mathrm{keV})
$$


to determine

$$
r_{N_{1}}=10^{-10}
$$

Finally, we assume the linear seesaw dominates the neutrino mass matrix. We further consider the simple case that the Yukawa couplings $h_{\nu}$ are symmetric, i.e. $h_{\nu}=h_{\nu}^{T}$. In this case, it is easy to determine $h_{\nu}$ by

$$
h_{\nu}=-\frac{\left\langle\chi_{R}\right\rangle}{2\langle\varphi\rangle\left\langle\chi_{L}\right\rangle} U_{\mathrm{PMNS}}^{*} \operatorname{diag}\left\{m_{1}, m_{2}, m_{3}\right\} U_{\mathrm{PMNS}}^{\dagger}
$$

Here $U_{\mathrm{PMNS}}$ is the Pontecorvo-Maki-Nakagawa-Sakata
24] (PMNS) leptonic mixing matrix while $m_{1,2,3}$ are the eigenvalues of the neutrino mass matrix. Currently the neutrino oscillation data have precisely measured the two neutrino mass squared differences [26],

$$
\begin{gathered}
\Delta m_{21}^{2}=m_{2}^{2}-m_{1}^{2}=7.65_{-0.20}^{+0.23} \times 10^{-5} \mathrm{eV}^{2} \\
\left|\Delta m_{31}^{2}\right|=\left|m_{3}^{2}-m_{1}^{2}\right|=2.40_{-0.11}^{+0.12} \times 10^{-3} \mathrm{eV}^{2}
\end{gathered}
$$

As for the PMNS matrix, it is consistent with the tribimaximal mixing [25],

$$
U_{\mathrm{PMNS}}=\left(\begin{array}{rrl}
\sqrt{\frac{2}{3}} & \sqrt{\frac{1}{3}} & 0 \\
-\sqrt{\frac{1}{6}} & \sqrt{\frac{1}{3}} & \sqrt{\frac{1}{2}} \\
\sqrt{\frac{1}{6}} & -\sqrt{\frac{1}{3}} & \sqrt{\frac{1}{2}}
\end{array}\right) \operatorname{diag}\left\{e^{i \frac{\alpha_{1}}{2}}, e^{i \frac{\alpha_{2}}{2}}, 1\right\},
$$

with which we can derive

$$
\begin{aligned}
& h_{\nu}=-\frac{\left\langle\chi_{R}\right\rangle}{2\langle\varphi\rangle\left\langle\chi_{L}\right\rangle}\left(\begin{array}{rr}
\frac{2}{3} \tilde{m}_{1}+\frac{1}{3} \tilde{m}_{2} & -\frac{1}{3} \tilde{m}_{1}+\frac{2}{3} \tilde{m} \\
-\frac{1}{3} \tilde{m}_{1}+\frac{2}{3} \tilde{m}_{2} & -\frac{1}{6} \tilde{m}_{1}+\frac{1}{3} \tilde{m} \\
\frac{1}{3} \tilde{m}_{1}-\frac{1}{3} \tilde{m}_{2} & -\frac{1}{6} \tilde{m}_{1}-\frac{1}{3} \tilde{m}
\end{array}\right. \\
& \text { For demonstration, let's focus on the normal hiera } \\
& \text { neutrinos, i.e. } \\
& \qquad m_{1}=0, \quad m_{2}=\sqrt{\Delta m_{21}^{2}}, \quad m_{3}=\sqrt{\left|\Delta m_{31}^{2}\right|} .
\end{aligned}
$$

It is easy to check that the linear seesaw dominates the neutrino mass matrix with the above parameter choice. We also can fix the parameters (22) to be

$$
K_{N_{1}^{+}} \simeq K_{N_{1}^{-}}=242 \text {. }
$$

By further inputting the $\mathrm{CP}$ phase

$$
\sin \frac{h_{\nu_{e 1}}^{*} h_{\xi_{e 1}}}{\left|h_{\nu_{e 1}} h_{\xi_{e 1}}\right|}=\sin \alpha_{2}=-0.13
$$

the CP asymmetries (18) can also be determined,

$$
\varepsilon_{N_{1}^{+}} \simeq \varepsilon_{N_{1}^{-}}=-2.99 \times 10^{-5} .
$$

In consequence, the final baryon asymmetry should arrive at

$$
\eta_{B}=0.886 \times 10^{-10},
$$

$$
\left.\begin{array}{l}
\frac{1}{3} \tilde{m}_{1}-\frac{1}{3} \tilde{m}_{2} \\
-\frac{1}{6} \tilde{m}_{1}-\frac{1}{3} \tilde{m}_{2}+\frac{1}{3} \tilde{m}_{3} \\
\frac{1}{6} \tilde{m}_{1}+\frac{1}{3} \tilde{m}_{2}+\frac{1}{3} \tilde{m}_{3}
\end{array}\right) \quad \text { with } \quad\left\{\begin{array}{l}
\tilde{m}_{1}=m_{1} e^{-i \alpha_{1}} \\
\tilde{m}_{2}=m_{2} e^{-i \alpha_{2}},(40) \\
\tilde{m}_{3}=m_{3} .
\end{array}\right.
$$

which is consistent with the five-year observations of the WMAP collaboration [27],

$$
\begin{aligned}
\eta_{B} & =\frac{1}{7.04} \times(6.225 \pm 0.170) \times 10^{-10} \\
& =(0.884 \pm 0.024) \times 10^{-10} .
\end{aligned}
$$

\section{LEPTOGENESIS WITH DOUBLE SEESAW}

We now discuss the case with $f\left\langle\chi_{R}\right\rangle \gg m_{\xi}$. In this case, the mass terms (8) can be diagonalized into two blocks, i.e.

$$
\mathcal{L} \supset-\frac{1}{2} m_{N} \bar{\nu}_{R}^{c} \nu_{R}-\frac{1}{2} m_{\xi} \bar{\xi}_{R}^{c} \xi_{R}+\text { H.c. . }
$$

Here the Majorana mass matrix of the right-handed neutrinos is a seesaw solution,

$$
m_{N}=-f^{*} \frac{\left\langle\chi_{R}\right\rangle^{2}}{m_{\xi}^{\dagger}} f^{\dagger}
$$


which can be of the order of $m_{N}=\mathcal{O}\left(10^{3}-10^{10} \mathrm{GeV}\right)$ for $m_{\xi}=\mathcal{O}\left(10^{16}-10^{19} \mathrm{GeV}\right)$ and $f\left\langle\chi_{R}\right\rangle=\mathcal{O}\left(10^{12}-\right.$ $10^{13} \mathrm{GeV}$ ). The Yukawa interaction (6) can thus realize the leptogenesis, with or without resonant effect, in the traditional way. The right-handed neutrinos are much lighter than the right-handed gauge bosons so that their gauge interactions can be decoupled naturally for the outof-equilibrium condition.

The right-handed neutrinos and the singlet fermions can both contribute to the neutrino masses,

$$
\mathcal{L} \supset-\frac{1}{2} \bar{\nu}_{L} m_{\nu} \nu_{L}^{c}+\text { H.c. . }
$$

with

$$
\begin{aligned}
m_{\nu} & \simeq h_{\nu} \frac{1}{f^{*}} m_{\xi} \frac{1}{f^{\dagger}} h_{\nu}^{T} \frac{\langle\varphi\rangle^{2}}{\left\langle\chi_{R}\right\rangle^{2}}-\left(h_{\nu}+h_{\nu}^{T}\right) \frac{\langle\varphi\rangle\left\langle\chi_{L}\right\rangle}{\left\langle\chi_{R}\right\rangle} \\
& =-h_{\nu} \frac{\langle\varphi\rangle^{2}}{m_{N}} h_{\nu}^{T}-\left(h_{\nu}+h_{\nu}^{T}\right) \frac{\langle\varphi\rangle\left\langle\chi_{L}\right\rangle}{\left\langle\chi_{R}\right\rangle}
\end{aligned}
$$

The first term is usually called the double seesaw [18] since there are two seesaw steps generating the small neutrino masses. The neutrino mass term can be conveniently expressed by

$$
m_{\nu}=m_{\nu}^{\text {Double }}+m_{\nu}^{\text {Linear }} .
$$

The linear seesaw $m_{\nu}^{\text {Linear }}$ could be comparable to the double seesaw $m_{\nu}^{\text {Double }}$. This implies we could relax the constraint on the CP asymmetry in the decays of the right-handed neutrinos from the neutrino masses if there is a cancellation between $m_{\nu}^{\text {Double }}$ and $m_{\nu}^{\text {Linear }}$.

\section{SUMMARY}

In summary we have discussed the realization of leptogenesis and seesaw by adding three singlet fermions with Majorana masses in the original left-right symmetric model with doublet and bi-doublet Higgs scalars. Depending on the size of the Majorana masses of the singlet fermions, the leptogenesis can be realized in different seesaw scenarios. If the Majorana masses are small, the right-handed neutrinos and the singlet fermions can form three pairs of quasi-degenerate Majorana fermions to accommodate the resonant leptogenesis at the $\mathrm{TeV}$ scale naturally. The neutrino masses are dominated by the linear seesaw, although the inverse seesaw also exists. In the other limiting case, where the singlet fermions are very heavy, the right-handed neutrinos can obtain Majorana masses through the seesaw contribution from the singlet fermions and then give a traditional picture of leptogenesis.

Acknowledgement: PHG thanks Manfred Lindner for hospitality at Max-Planck-Institut für Kernphysik and thanks the Alexander von Humboldt Foundation for financial support.
[1] P. Minkowski, Phys. Lett. B 67, 421 (1977).

[2] T. Yanagida, in Proc. of the Workshop on Unified Theory and the Baryon Number of the Universe, ed. O. Sawada and A. Sugamoto (KEK, Tsukuba, 1979), p. 95.

[3] M. Gell-Mann, P. Ramond, and R. Slansky, in Supergravity, ed. F. van Nieuwenhuizen and D. Freedman (North Holland, Amsterdam, 1979), p. 315.

[4] S.L. Glashow, in Quarks and Leptons, ed. M. Lévy et al. (Plenum, New York, 1980), p. 707.

[5] R.N. Mohapatra and G. Senjanović, Phys. Rev. Lett. 44, 912 (1980).

[6] M. Magg and C. Wetterich, Phys. Lett. B 94, 61 (1980); J. Schechter and J.W.F. Valle, Phys. Rev. D 22, 2227 (1980); T.P. Cheng and L.F. Li, Phys. Rev. D 22, 2860 (1980); G. Lazarides, Q. Shafi, and C. Wetterich, Nucl. Phys. B 181, 287 (1981); R.N. Mohapatra and G. Senjanović, Phys. Rev. D 23, 165 (1981).

[7] J.C. Pati and A. Salam, Phys. Rev. D 10, 275 (1974); R.N. Mohapatra and J.C. Pati, Phys. Rev. D 11, 566 (1975); R.N. Mohapatra and J.C. Pati, Phys. Rev. D 11, 2558 (1975); R.N. Mohapatra and G. Senjanović, Phys. Rev. D 12, 1502 (1975).

[8] M. Fukugita and T. Yanagida, Phys. Lett. B 174, 45 (1986).

[9] R.N. Mohapatra and X. Zhang, Phys. Rev. D 46, 5331 (1992).
[10] E Ma and U. Sarkar, Phys. Rev. Lett. 80, 5716 (1998).

[11] M. Flanz, E.A. Paschos, and U. Sarkar, Phys. Lett. B 345, 248 (1995); M. Flanz, E.A. Paschos, U. Sarkar, and J. Weiss, Phys. Lett. B 389, 693 (1996); L. Covi, E. Roulet, and F. Vissani, Phys. Lett. B 384, 169 (1996).

[12] A. Pilaftsis, Phys. Rev. D 56, 5431 (1997).

[13] T. Hambye and G. Senjanovic, Phys. Lett. B 582, 73 (2004); S. Antusch and S.F. King, Phys. Lett. B 597, 199 (2004).

[14] V.A. Kuzmin, V.A. Rubakov, and M.E. Shaposhnikov, Phys. Lett. B 155, 36 (1985).

[15] P. Langacker, R.D. Peccei, and T. Yanagida, Mod. Phys. Lett. A 1, 541 (1986); M.A. Luty, Phys. Rev. D 45, 455 (1992).

[16] A. Pilaftsis, Phys. Rev. D 60105023 (1999); P.H. Gu, Phys. Rev. D 81, 073002 (2010).

[17] S. Davidson and A. Ibarra, Phys. Lett. B 535, 25 (2002); W. Buchmüller, P. Di Bari, and M. Plümacher, Nucl. Phys. B 665, 445 (2003). For a review, W. Buchmüller, R.D. Peccei, and T. Yanagida, Ann. Rev. Nucl. Part. Sci. 55, 311 (2005).

[18] R.N. Mohapatra, Phys. Rev. Lett. 56, 561 (1986).

[19] R.N. Mohapatra and J.W.F. Valle, Phys. Rev. D 34, 1642 (1986); M.C. Gonzalez-Garcia and J.W.F. Valle, Phys. Lett. B 216, 360 (1989).

[20] S.M. Barr, Phys. Rev. Lett. 92, 101601 (2004). 
[21] S.F. King and T. Yanagida, Prog. Theor. Phys. 114, 1035 (2006).

[22] C.H. Albright and S.M. Barr, Phys. Rev. D 69, 073010 (2004).

[23] E.W. Kolb and M.S. Turner, The Early Universe, Addison-Wesley, 1990.

[24] Z. Maki, M. Nakagawa, and S. Sakata, Prog. Theor. Phys. 28, 870 (1962); B. Pontecorvo, Sov. Phys. JETP 26, 984
(1968), Zh. Eksp. Teor. Fiz. 53, 1717 (1967).

[25] P.F. Harrison, D.H. Perkins, and W.G. Scott, Phys. Lett. B 530, 167 (2002).

[26] T. Schwetz, M.A. Tórtola, and J.W.F. Valle, New J. Phys. 10, 113011 (2008).

[27] J. Dunkley et al., [WMAP Collaboration], Astrophys. J. Suppl. 180, 306 (2009). 